\title{
Measuring irreversible dynamics of a quantum harmonic oscillator
}

\author{
Giovanna Morigi, ${ }^{1}$ Enrique Solano, ${ }^{1,2}$ Berthold-Georg Englert, ${ }^{1}$ and Herbert Walther ${ }^{1}$ \\ ${ }^{1}$ Max-Planck-Institut für Quantenoptik, Hans-Kopfermann-Strasse 1, 85748 Garching, Germany \\ ${ }^{2}$ Sección Física, Departamento de Ciencias, Pontificia Universidad Católica del Perú, Apartado 1761, Lima, Peru
}

(Received 20 August 2001; published 10 April 2002)

\begin{abstract}
We show that the unitary evolution of a harmonic oscillator coupled to a two-level system can be undone by a suitable manipulation of the two-level system-more specifically, by a quasi-instantaneous phase change. This enables us to isolate the dissipative evolution to which the oscillator may be exposed in addition. With this method we study the decoherence time of a photon mode in cavity QED, and that of the quantized harmonic motion of trapped ions. We comment on the relation to spin echoes and multipath interferometry.
\end{abstract}

DOI: 10.1103/PhysRevA.65.040102

PACS number(s): 03.65.Yz, 32.80.Qk, 42.50.Ct

Decoherence is currently under investigation theoretically and experimentally [1], thereby searching for the boundary between the quantum and classical realms [2,3]. From the point of view of technical applications, scalability of coherent control-like that needed in a quantum computer-is being pursued intensively and is limited mainly by the presence of decoherence $[1,4]$. With the tools of quantum optics, it has become possible to create quantum states of high purity [4] and to monitor their decay [5,6]. The measurement of a quantum state, however, can introduce additional coherence destruction, caused by the nonunitarity of the state reduction. Methods like quantum nondemolition measurements [7], partially solving this problem, have been implemented for studying nonclassical states of a harmonic oscillator and their decoherence [5]. Nevertheless, the decoherence of arbitrary initial states of the quantum oscillator deserves further studies.

In this contribution, we propose a method for measuring the decoherence time of a harmonic oscillator prepared in an arbitrary initial state by suitably coupling it to a stable twolevel system (TLS), whose final level statistics carry the information of the oscillator decay. The composite system evolves following a Jaynes-Cummings (JC) interaction [8] for a total duration $T$. At an intermediate instant, after time $\tau$ has elapsed, the JC evolution is interrupted by a phase kick, that is, a fast unitary operation applied to the TLS that introduces a relative phase between the levels. We will show that, as a consequence of the phase kick, the coherent dynamics is effectively reversed, mimicking a time-reversal operation [9-11]. When $\tau=T / 2$, the final state of the composite system matches the initial one, provided that the evolution is unitary. Any mismatch represents a measurement of changes in the oscillator state due to external actions and an estimation of the time in which decoherence occurs.

This method allows us to separate the irreversible decay of the oscillator state from the unitary JC dynamics. The scheme can be easily implemented in two representative scenarios in quantum optics, ion traps and cavity QED, for studying the irreversible decay of the quantized motion or the electromagnetic field, respectively. In cavity QED, the harmonic oscillator is a mode of the quantized radiation field, coupled to a resonant electronic transition of atoms sent through the resonator and undergoing JC dynamics [12]. In ion traps, the center-of-mass motion of the trapped ion is harmonic, and it couples to an internal atomic transition when the ion is irradiated by a laser [13]. We discuss below how the decay of these oscillators can be studied by means of the proposed scheme.

Let us consider the resonant interaction between a TLS, with lower state $|\mathrm{g}\rangle$ and upper state $|\mathrm{e}\rangle$, and a harmonic oscillator, with creation and annihilation operators $a^{\dagger}$ and $a$, respectively. The eigenstates of the number operator $a^{\dagger} a$ are denoted by $|n\rangle$ with $n=0,1,2, \ldots$ The transition energy of the TLS is $\hbar \omega$, equal to the energy of the oscillator quanta. The coherent dynamics is described by the Hamiltonian

$$
H(t)=H_{\mathrm{JC}}+H_{\text {kick }}=H_{0}+H_{\text {int }}+H_{\text {kick }} .
$$

Here, $H_{\mathrm{JC}}$ describes the JC dynamics, composed of the free Hamiltonian

$$
H_{0}=\hbar \omega\left(\sigma^{\dagger} \sigma+a^{\dagger} a\right)
$$

with $\sigma=|\mathrm{g}\rangle\left\langle\mathrm{e}\left|, \sigma^{\dagger}=\right| \mathrm{e}\right\rangle\langle\mathrm{g}|$, and of the interaction term

$$
H_{\text {int }}=\hbar g\left(\sigma^{\dagger} a+\sigma a^{\dagger}\right),
$$

where $g$ is the coupling constant. $H_{\text {kick }}$ generates the instantaneous phase kick at $t=\tau$ and is defined as

$$
H_{\mathrm{kick}}=\hbar \pi \sigma \sigma^{\dagger} \delta(t-\tau) .
$$

The effect of the kick alone is thus given by

$$
U_{\text {kick }}=\exp \left(-\frac{i}{\hbar} \int_{\tau-0}^{\tau+0} d t H(t)=\sigma^{\dagger} \sigma-\sigma \sigma^{\dagger}=\sigma_{z} .\right.
$$

After the duration $T$, the evolution operator for the whole process is then

$$
\begin{aligned}
U_{\tau}(T) & =e^{-(i / \hbar) H_{\mathrm{JC}}(T-\tau)} U_{\mathrm{kick}} e^{-(i / \hbar) H_{\mathrm{JC}} \tau} \\
& =\sigma_{z} \exp \left[\frac{i}{\hbar}\left(H_{0}+\frac{2 \tau-T}{T} H_{\mathrm{int}}\right) T\right],
\end{aligned}
$$

where the second line exploits $\left[H_{0}, H_{\text {int }}\right]=0$ and $\left\{H_{\text {int }}, \sigma_{z}\right\}$ $=0$. So the net evolution amounts to propagation governed by a JC coupling of the effective strength 

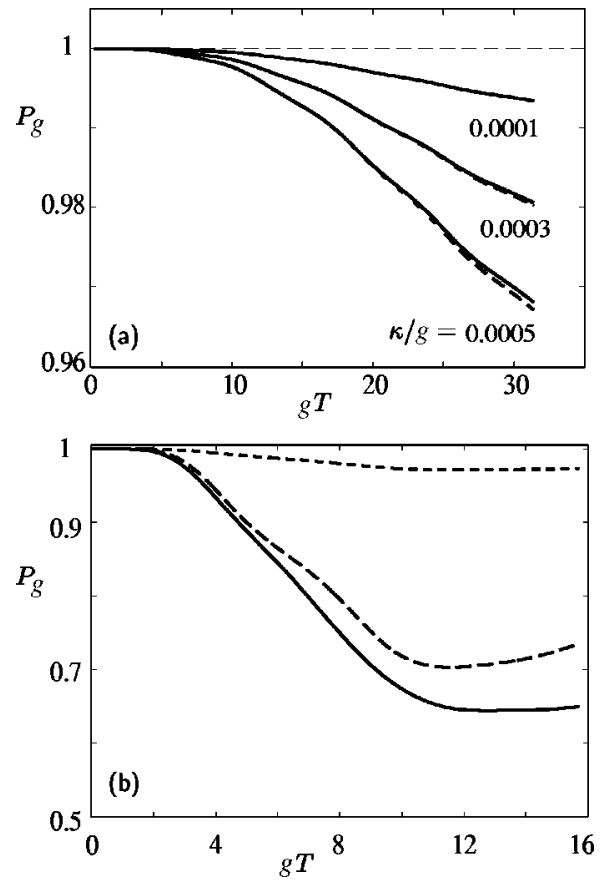

FIG. 1. (a) Numerical values [solid line, Eq. (8)] and approximate analytical values [dashed line, Eq. (10)] of $P_{\mathrm{g}}$ as a function of $g T$ for different ratios $\kappa / g$ and the field initially in the number state $|3\rangle$. (b) Numerical values of $P_{\mathrm{g}}$ for $\kappa=0.05 \mathrm{~g}$ and initial cavity states $(|2\rangle+i|3\rangle) / \sqrt{2}$ (solid line), $|2\rangle$ (dashed line), coherent state $|\alpha\rangle$ with $\alpha=\exp (i \pi / 4) / \sqrt{2}$ (dotted line).

$$
g_{\text {eff }}=\frac{2 \tau-T}{T} g
$$

for time $T$, followed by the phase kick. Since the probability of finding the TLS in $|\mathrm{g}\rangle$ or $|\mathrm{e}\rangle$ is measured eventually, this final phase kick can in fact be ignored. For $\tau=T / 2$, the effective interaction term in Eq. (6) vanishes and the final state is just the freely evolved initial state (except for the irrelevant phase kick). So, if the initial state were $|g\rangle \otimes\left|\psi_{\text {osc }}\right\rangle$, for example, the TLS would always be measured in the ground state after the interaction. The effect of the kick consists thus in symmetrizing the JC evolution [10], which is somewhat reminiscent of spin-echo techniques [14], where the evolution of spin- $\frac{1}{2}$ particles interacting with a classical field is undone. We remark that, with this scheme, the external action could come from any other coherent or even incoherent process, but not from the probing TLS. Nevertheless, it is its final statistics that carry the information about the effect of the environment on the oscillator.

We now turn to the application of this scheme to the particular scenario of cavity QED. The quantized electromagnetic field in a cavity is probed by atoms that fly through the resonator and whose dipole transition couples resonantly to a privileged field mode, undergoing the JC interaction described by $H_{\mathrm{JC}}$ in Eq. (1). The phase kick could be implemented by a laser that quasiresonantly couples the ground state of the atom to a third atomic level, thereby realizing a fast $2 \pi$ pulse [11]. We consider the proposed scheme for measuring the decoherence time of the cavity field, coupled to a Markovian bath at zero temperature [12]. We assume that the atomic transition is not subject to dissipation by itself on the relevant time scale. The initial state $\rho(0)$ of the joint atom-field system is then evolved into the final state in accordance with

$$
\rho(T)=e^{L(T-\tau)} K e^{L \tau} \rho(0),
$$

where $K \rho=U_{\text {kick }} \rho U_{\text {kick }}^{-1}=\sigma_{z} \rho \sigma_{z}$ is the effect of the kick at time $\tau$, and the Liouville operator $L$ is given by

$$
L \rho=-\frac{i}{\hbar}\left[H_{\mathrm{JC}}, \rho\right]+\kappa\left(a \rho a^{\dagger}-\frac{1}{2} a^{\dagger} a \rho-\frac{1}{2} \rho a^{\dagger} a\right),
$$

where $\kappa$ is the field decay rate. This particular choice of the nonunitary part of $L$ is taken for simplicity, but the method works just as well for other cases. An explicit form of $P_{\mathrm{g}}$, the probability for measuring the atom at the exit in state $|\mathrm{g}\rangle$, can be obtained from Eq. (8) in the limits $\kappa T \ll 1$ and $\kappa / g$ $\ll 1$. At first order in these parameters, for $\tau=T / 2$ and $\rho(0)$ $=|\mathrm{g}\rangle\left\langle\mathrm{g}\left|\otimes \Sigma_{n, m} \rho_{n m}\right| n\right\rangle\langle m|$, it is

$$
\begin{aligned}
P_{\mathrm{g}}= & 1-\sum_{n=2}^{\infty} \rho_{n, n}\left\{\frac{\kappa T}{4}(2 n-1)\right. \\
& +\kappa \frac{\sin (g T \sqrt{n})}{4 g \sqrt{n}}-\kappa \frac{\sin (g T \sqrt{n-1})}{4 g \sqrt{n-1}} \\
& -\frac{\kappa}{4 g}[\sqrt{n}(4 n-3) \sin (g T \sqrt{n}) \cos (g T \sqrt{n-1}) \\
& -\sqrt{n-1}(4 n-1) \sin (g T \sqrt{n-1}) \cos (g T \sqrt{n})]\} .
\end{aligned}
$$

The effect of the decay is evident, causing deviations of $P_{\mathrm{g}}$ from unity. These deviations are oscillatory functions of the duration $T$, resulting from the JC dynamics characterizing the system evolution. Note that in Eq. (10) the summation starts at $n=2$. In fact, for $\rho_{n n}=\delta_{n, 1}$ one has $P_{\mathrm{g}}=1$ in this perturbative limit, the deviations appearing at second order [15].

In Fig. 1, we plot $P_{\mathrm{g}}$ as a function of the duration $T$. Figure 1(a) compares the full numerical evaluation of Eq. (8) with the approximation (10) for different decay rates $\kappa$. In Fig. 1(b), the curves are plotted for different initial states. Note that for $\kappa T \gg 1$ the cavity state tends to the vacuum, and the probability of counting the atoms in the ground state approaches unity again. How big the deviation from $P_{\mathrm{g}}=1$ should be for considering that the system has decohered is a question that will depend on each particular case or use.

The decay of the field phase can be studied by adding to the proposed scheme a Ramsey zone at the exit of the cavity, which performs the unitary transformation $\exp \left[-i \phi\left(\sigma e^{i \zeta}\right.\right.$ $\left.\left.+\sigma^{\dagger} e^{-i \zeta}\right)\right]$. In this way, the Ramsey zone and the detectors constitute a field-phase-sensitive device [16]. When only the coherent atom-field interaction takes place, we have $P_{\mathrm{g}}$ $=\cos ^{2} \phi$, independent of the initial state of the field, and the decay of the field phase is measured through the deviations from this value. In Fig. 2, we plot the phase sensitive probability $P_{\mathrm{g}}$, after the Ramsey zone with $\phi=\pi / 4, \zeta=0$, as a 


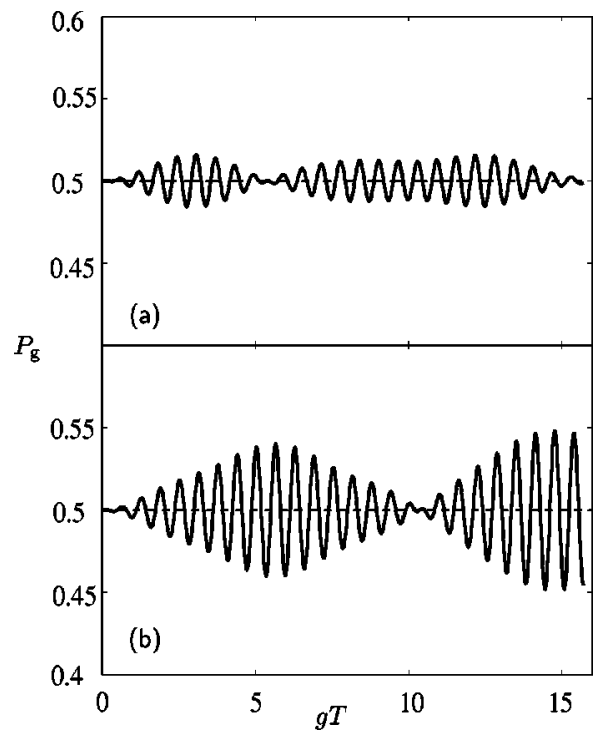

FIG. 2. Phase sensitive $P_{\mathrm{g}}$ as a function of $g T$ for $\kappa=0.05 g$ and $\omega=10 g$. The initial field states are (a) $(|2\rangle+i|3\rangle) / \sqrt{2}$ and (b) coherent state $|\alpha\rangle$ with $\alpha=\exp (i \pi / 4) / \sqrt{2}$. In both plots, the constant $P_{\mathrm{g}}=0.5$ of an initial Fock state is plotted for reference.

function of the total interaction time for different initial field states. Both signals contain a high frequency modulation at the field frequency, chosen here for convenience as $\omega=10 \mathrm{~g}$. For reference, we plot in both figures the curve corresponding to an initial state characterized only by a diagonal density matrix (dashed line)—a Fock state, say.

We now study the application of the phase-kick scheme in ion traps, where a JC dynamics between the internal transition of the ions and its center-of-mass motion is implemented by a laser. Now, the Hamilton operator (1) applies in the reference frame rotating at the laser frequency. The atomic phase kick can be realized here in a similar way as in the cavity QED case. Provided that the spontaneous emission from the atom can be neglected and that the motion is coupled to a Markovian bath at zero temperature, the evolution of the density matrix of the composite system is again given by Eq. (8), and the results reported above for the atomcavity case are recovered. In the ion-trap situation, however, the phase kick is more easily implemented than in cavity QED. For example, we can switch off the laser system, after a fast initial coherent JC evolution and before the phase kick, and so interrupt the interaction during a convenient period of time $T_{\text {free }}$. By continuing with the phase kick and a fast final coherent JC evolution, the experiment is completed.

This variation of our scheme has features in common with Ramsey interferometry [17]. One could say that, during the initial short interaction time, interferometric paths are created in the ion-motion Hilbert space and that, after suffering "dephasing" during time $T_{\text {free, }}$, these paths are recombined into the initial $g_{\text {eff }}=0$ superposition. With the aid of an arbitrary phase kick, instead of the fixed $2 \pi$ kick, we create a modulated output that is reminiscent of an interferometer pattern. This variation could also be implemented in the atom-cavity system, but the experimental setup would be much more demanding.
In general, the scheme can be applied to studying decoherence in the collective motion of a chain of $N$ ions [18], i.e., a linear crystal of ions, achieved in linear ion traps at sufficiently low temperature. In this limit, the chain motion along its main axis is described by the collective excitations of its $N$ normal modes with frequencies $\nu_{1}, \ldots, \nu_{N}[19]$. A dynamics of the JC type can be designed between the $j$ th ion internal state and the $\beta$ th mode of the crystal, by laser irradiation analogous to the one-ion case. Then, the total Hamiltonian describing the interaction is given by $H_{\mathrm{JC}}^{(j, \beta)}=H_{0}^{(j, \beta)}$ $+H_{\text {int }}^{(j, \beta)}$ with

$$
H_{0}^{(j, \beta)}=\hbar \nu_{\beta}\left(\sigma_{j}^{\dagger} \sigma_{j}+a_{\beta}^{\dagger} a_{\beta}\right)
$$

and

$$
H_{\mathrm{int}}^{(j, \beta)}=\hbar g_{j, \beta}\left(\sigma_{j}^{\dagger} a_{\beta}+\sigma_{j} a_{\beta}^{\dagger}\right)
$$

where $g_{j, \beta}$ is the Rabi frequency for ion $j$ and mode $\beta, a_{\beta}$, and $a_{\beta}^{\dagger}$ are the annihilation and creation operators of a vibrational quantum $\hbar \nu_{\beta}$, while $\nu_{\beta}$ is the detuning of the laser from the atomic transition and equal to the frequency of the addressed mode. The dynamics then follows the lines of that described for a single ion, except that here the $\sigma_{z}$ kick is a laser pulse applied to ion $j$. In this way, we are able to study the irreversible decay of a chosen collective mode. Similarly, it is possible to measure the irreversible loss of coherence of an arbitrary motional state involving all modes. One would need to pair the ions with the modes, so that the corresponding Hamiltonian has the form $H=\Sigma_{(j, \beta)} H_{\mathrm{JC}}^{(j, \beta)}$, a sum over these pairs. One could then measure the decoherence time of each mode, and also of entangled states between different normal modes.

Here are some remarks on the experimental feasibility of the proposed scheme in relation to the systems discussed before. First, we note that the phase kick is crucial for the whole scheme, but it is not necessary to realize the ideal kick of Eq. (5). Indeed, the scheme is rather robust against experimental imperfections that would amount to multiplying $H_{\text {kick }}$ of Eq. (4) by $1+\epsilon$. In particular, even with an error as large as $|\epsilon| \lesssim 7 \%$, we would still cancel more than $99 \%$ of the JC dynamics.

In the case of cavity QED, our scheme could be implemented in the microwave and optical regime. For a test of the decoherence time associated with the population and the phase of the intracavity field, convenient ratios $\kappa / g \leqslant 1$ can be found in both regimes. In either case, atomic decay is irrelevant in a wide range of interaction times. Furthermore, the kick could be implemented by introducing a sheet of light - transversal to the mode of interest and crossed by the atomic trajectory at the center of the cavity-by shining a laser through a hole drilled at the side of the cavity (closed cavity), or by focusing laser beams (open cavity). Note that, for measuring decoherence in atom-cavity systems, a quality factor that is not too large may be desirable. Further, the use of an external kick for testing this scheme is not necessary, if the field mode in question changes sign at the center of the cavity. In this case, the odd spatial symmetry of the field 
naturally undoes the JC dynamics, producing the same effect as the phase kick for the fundamental mode.

Ion traps appear more fitting for testing and scaling the present scheme. For example, the atomic decay can be neglected by suitably coupling either a long-lived transition or two internal metastable states with a two-photon coherent process. Also, JC (or anti-JC) dynamics like that discussed here as well as pulses of well defined shape and duration are currently realized. Individual ions of a chain cannot be addressed by a laser in all experiments, but this is not necessary for the present application. It is always possible to illuminate the chain of ions homogeneously and, by giving an equally homogeneous phase kick, the JC dynamics could be "time reversed" and tested by measuring the global atomic ground state. In this case, we replace $\sigma$ by $\Sigma_{j} \sigma_{j}$ in Eq. (12), and the phase-kick operator $\sigma_{z}$ by $\Pi_{j} \sigma_{z}^{j}$.

In summary, we have discussed a method for measuring the decoherence time of a harmonic oscillator by coupling it to a two-level system and detecting its level statistics. The method is based on a phase kick applied to the two-level system during its interaction with the oscillator, thereby undoing the coherent interaction and isolating the effect of any external disturbance affecting the oscillator. We have discussed applications of the scheme for measuring decoherence times of the quantized field mode in cavity QED experiments and of the quantized motion of trapped atoms in harmonic traps. We think that the proposed scheme represents a useful tool for measuring decoherence times in different physical systems undergoing JC-like interactions. As a general feature, this method is sensitive to any deviation from the expected JC dynamics and could also be used, for instance, for measuring corrections to the rotating wave approximation, or for studying the anharmonic coupling among the normal modes of a chain of ions, or the influence of spectator atoms and spectator modes.

This work was partly supported by the European Commission (TMR networks ERB-FMRX-CT96-0077 and ERBFMRX-CT96-0087).
[1] See for example, Decoherence: Theoretical, Experimental and Conceptual Problems, Vol. 538 of Lecture Notes in Physics, edited by P. Blanchard, D. Giulini, E. Joos, C. Kiefer, and I.-O. Stamatescu (Springer, Berlin, 2000).

[2] P. Bertet, S. Osnaghi, A. Rauschenbeutel, G. Nogues, A. Auffeves, M. Brune, J.M. Raimond, and S. Haroche, Nature (London) 411, 166 (2001).

[3] M. Arndt, O. Nairz, J. Vos-Andreae, C. Keller, G. van der Zouw, and A. Zeilinger, Nature (London) 401, 680 (1999); M. Arndt and A. Zeilinger, Phys. B1. 56, 69 (2000).

[4] See, for example, The Physics of Quantum Information, edited by D. Bouwmeester, A. Ekert, and A. Zeilinger (Springer, Berlin, 2000).

[5] M. Brune, E. Hagley, J. Dreyer, X. Maitre, A. Maali, C. Wunderlich, J.M. Raimond, and S. Haroche, Phys. Rev. Lett. 77, 4887 (1996).

[6] C.J. Myatt, B.E. King, Q.A. Turchette, C.A. Sackett, D. Kielpinski, W.M. Itano, C. Monroe, and D.J. Wineland, Nature (London) 403, 269 (2000).

[7] V.B. Braginsky and F.Y. Khalili, in Quantum Measurement, edited by K.S. Thorne (Cambridge University Press, Cambridge, England, 1992).

[8] E.T. Jaynes and F.W. Cummings, Proc. IEEE 51, 89 (1963).

[9] Phase kicks of this sort are at the basis of schemes for inhibiting decoherence of a small system coupled to a bath. See, for example, L. Viola and S. Lloyd, Phys. Rev. A 58, 2733 (1998); D. Vitali and P. Tombesi, ibid. 59, 4178 (1999); L. Viola, E.
Knill, and S. Lloyd, Phys. Rev. Lett. 82, 2417 (1999); L. Viola, S. Lloyd, and E. Knill, ibid. 83, 4888 (1999).

[10] P. Zanardi, Phys. Lett. A 258, 77 (1999).

[11] See, for example, G.S. Agarwal, M.O. Scully, and H. Walther, Phys. Rev. Lett. 86, 4271 (2001).

[12] G. Raithel, C. Wagner, H. Walther, L.M. Narducci, and M.O. Scully, in Advances in Atomic, Molecular and Optical Physics, edited by P. Berman (Academic, New York, 1994), Suppl. 2.

[13] D.J. Wineland, C. Monroe, W.M. Itano, D. Leibfried, B.E. King, and D.M. Meekhof, J. Res. Natl. Inst. Stand. Technol. 103, 259 (1998).

[14] See, for example, L. Allen, and J.H. Eberly, Optical Resonance and Two-Level Atoms (Wiley, London, 1975).

[15] For the initial state $\rho(0)=|\mathrm{g}, 1\rangle\langle\mathrm{g}, 1|$, one can evaluate $P_{\mathrm{g}}$ exactly:

$$
P_{\mathrm{g}}=1-64 \frac{g^{2} \kappa^{2} e^{-\kappa T / 2}}{\left(16 g^{2}-\kappa^{2}\right)^{2}} \sin ^{4}\left(\frac{1}{8} \sqrt{16 g^{2}-\kappa^{2}} T\right),
$$

which shows that the effect of the decay is $O\left(\kappa^{2} / g^{2}\right)$.

[16] B.-G. Englert, T. Gantsog, A. Schenzle, C. Wagner, and H. Walther, Phys. Rev. A 53, 4386 (1996).

[17] N.F. Ramsey, Molecular Beams (Oxford University Press, New York, 1985).

[18] E. Solano, R.L. de Matos Filho, and N. Zagury, Phys. Rev. Lett. 87, 060402 (2001).

[19] D.F.V. James, Appl. Phys. B: Lasers Opt. 66, 181 (1998). 